\title{
Simulation of dynamic response of projectile and granular target
}

\author{
Kinya Ogawa ${ }^{1, \text { a }}$, Shin-nosuke Takeda ${ }^{2}$, Hidetoshi Kobayashi ${ }^{2}$, and Kenichi Tanigaki ${ }^{2}$ \\ ${ }^{1}$ Institute of Space Dynamics, Ondoyama, Narutaki, Ukyo-KU, Kyoto 616-8255, Japan \\ 2 School of Engineering Science, Osaka University 1-3 Machikaneyama-cho, Toyonaka, Osaka 560-8531, Japan
}

\begin{abstract}
In the present study, the three-dimensional elastic discrete element method (DEM) and the elastic finite element method (FEM) analysis were, respectively, applied to the randomly distributed identical spheres as a granular target and to the cylindrical projectiles in order to clarify dynamic response during penetration. In the target, it was found that highly densified region was formed just ahead of the projectile and began to propagate spherically at much higher velocity than that of projectile leaving relatively rarefied region. It was also found that the peak resistance during penetration was closely related to the initial formation of densified region and was expressed in terms of momentum change of target particles accelerated by the projectile. Stress wave propagation in the projectiles with various body lengths was investigated during penetration and was discussed in connection with dynamic response of target particles ahead of the projectile. Effect of mechanical properties of projectile on the peak resistance was also investigated and was understood in connection with dynamic behavior of target particles.
\end{abstract}

\section{Introduction}

Collision impact between continuous medium and granular materials such as sand, soil, and heterogeneous brittle aggregates is interesting subject and has been investigated over several decades. Resistance during penetration has been identified in experiments and a various formulations for the resistance versus penetration relations have been proposed [1,2]. Direct measurement of resistance has been also tried $[3,4]$. It would be the first and most fundamental approach to clarify dynamic characteristics in the problem based on elastic wave propagation theory. It is also necessary to find out effect of mechanical impedance of materials upon the dynamic behavior of subject.

In the present study the discrete element method together with the finite element method were applied to simulate the dynamic behaviors of penetration into granular target and the characteristics of the problem were fully discussed in connection with dynamic movement of particles together with dynamic response of projectile.

\section{Computational setup}

\subsection{Projectile}

Figure 1 shows a projectile and a target of granular medium. A projectile was a cylinder of $42 \mathrm{~mm}$ in diameter and three kinds of length with a flat end. Three dimensional elastic FEM model was applied to the projectile having Young' modulus of $69 \mathrm{GPa}$ and density of $3.8 \times 10^{3} \mathrm{Kg} / \mathrm{m}^{3}$ with Poisson's ratio of 0.3 . Initial velocity of the projectile was in the range of $60 \mathrm{~m} / \mathrm{s}$ to $240 \mathrm{~m} / \mathrm{s}$.

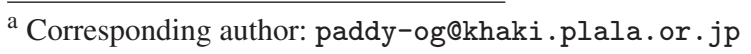

\subsection{Target}

The target was formed by randomly distributing identical spheres of $2.18 \mathrm{~mm}$ in diameter and of $3.76 \mathrm{~g} / \mathrm{cm}^{3}$ in density with Young's modulus of $310 \mathrm{GPa}$ into a spheroidal container of $218 \mathrm{~mm}$ in diameter and of $160 \mathrm{~mm}$ in depth as shown in the figure. Average packing density was changed from about $60 \%$ to $48 \%$. Elastic Discrete Element Method (DEM) was applied for the target.

Reactions between spheres were expressed by springs both in normal and tangential directions, together with a slider to represent static and kinetic frictions as shown in Fig. 2. Numerical simulations were carried out with time increment of $2.94 \times 10^{-8} \mathrm{~s}$.

\section{Results}

\subsection{Behavior of target}

To clarify deformation state in the particulate target we have focus our attention to the change of apparent packing density which may reflects to stress and strain in the sense of continuous mechanics. Figure 3 represents distribution of packing density caused by penetration of projectile at initial impact velocity of $120 \mathrm{~m} / \mathrm{s}$ and $240 \mathrm{~m} / \mathrm{s}$. Just after the impact, higher density region appears just ahead of the projectile, and then, at almost the peak resistance which will be explained in detail, the region spreads spherically and begins to propagate downward at much higher velocity than that of projectile leaving relatively rarefied region. This rarefied region is essentially caused by difference of velocities of the projectile and the high density region and such velocity difference would be decreased with the increase of initial impact velocity.

With increase of initial impact velocity, the high density region grows much clearly and tails up to just ahead of the projectile as seen in the figure.

This is an Open Access article distributed under the terms of the Creative Commons Attribution License 4.0, which permits unrestricted use, distribution, and reproduction in any medium, provided the original work is properly cited. 


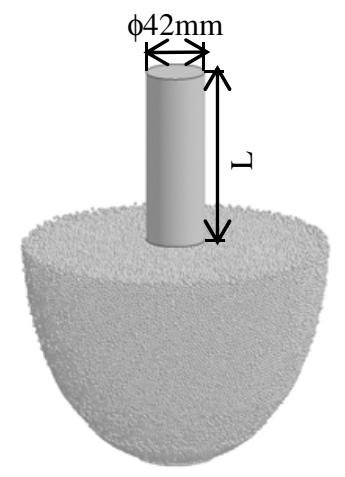

Figure 1. Target and flat-ended cylindrical projectile.

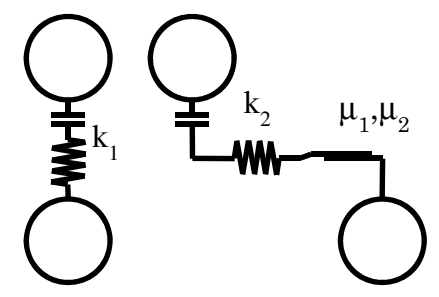

$$
\begin{aligned}
& \mathrm{k}_{\mathrm{i}} \text { : spring constant } \\
& \mu_{1} \text { : coefficient of static friction } \\
& \mu_{2} \text { : coefficient of kinetic friction }
\end{aligned}
$$

Figure 2. Mechanical model of granular medium with elastic springs and sliders to represent static and kinetic friction.
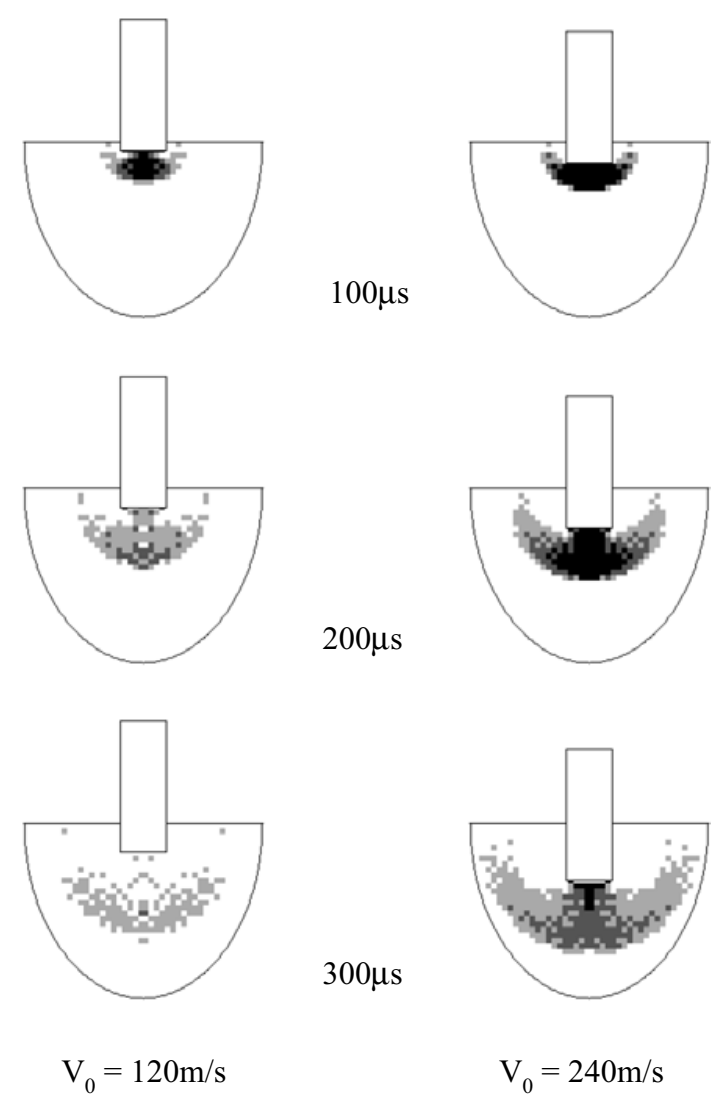

$\mathrm{V}_{0}=120 \mathrm{~m} / \mathrm{s}$

Figure 3. Distribution of density in target during penetration at different impact velocity.

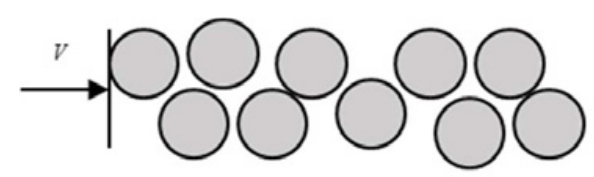

(a)

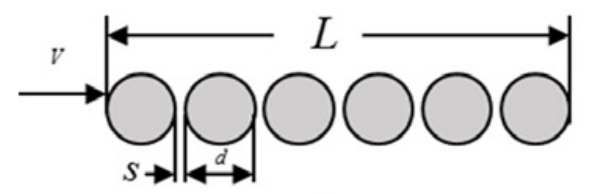

(b)

Figure 4. a), b) Illustration of arrangement of spheres.

The propagation velocity of such high density region was determined as follows. The location of the most distant particle which began to move at time $t$ in vertical direction is expressed by $(Y, r)$ and that at time $t+\Delta t$ is as $(Y+$ $\Delta Y, r+\Delta r)$, and then the propagation velocity, $C$, can be evaluated as

$$
C=(\Delta Y / \Delta t) \text {. }
$$

In the case of granular medium the elastic wave velocity depends on the density. Elastic wave propagation in randomly arranged particles with separations as illustrated in Fig. 4 a can be simplified by that in aligned particles with separations as shown in Fig. 4b. When the velocity, $V$, is applied to the left end particle, then it moves and hits its next neighbor particle and so on. Then we have the following relation for the elastic wave propagation;

$$
\frac{n d+(n-1) s}{C}=\frac{n d}{C_{0}}+\frac{(n-1) s}{V}
$$

where $C_{0}$ is the elastic wave velocity in the case of $s=0$, and $n$ is a number of particles in the length $L$. Equation (2) can be rewritten as follows.

$$
\frac{1}{C}=\alpha \frac{1}{C_{0}}+\beta \frac{1}{V}, \alpha=\frac{1}{(1+s / d)}, \beta=\frac{s / d}{(1+s / d)} .
$$

In Fig. 5, the propagation velocities at the peak resistance of projectile are plotted against the velocity of projectile following the Eq. (3) for different packing densities. The values of $s / d$ can be obtained from the slope for respective lines. Since the close packing density is given by $\pi \sqrt{2} / 6(1+s / d)^{-3}$, the estimated values are of $63 \%$, $59 \%, 54 \%$ and $44 \%$ which are slightly different from the used packing density of $60 \%, 57 \%, 53 \%$ and $48 \%$, respectively in the present simulation. The values of $C_{0}$ can be obtained by using the respective intercepts of lines together with the values of $s / d$, and are ranged from $500 \mathrm{~m} / \mathrm{s}$ to $530 \mathrm{~m} / \mathrm{s}$ almost corresponding to the value of $530 \mathrm{~m} / \mathrm{s}$ for rectangular lattice arrangement without separation [5].

\subsection{Wave propagation in a projectile}

Elastic wave propagation in the projectile was investigated and was exampled in Fig. 6. At the impact onto the 


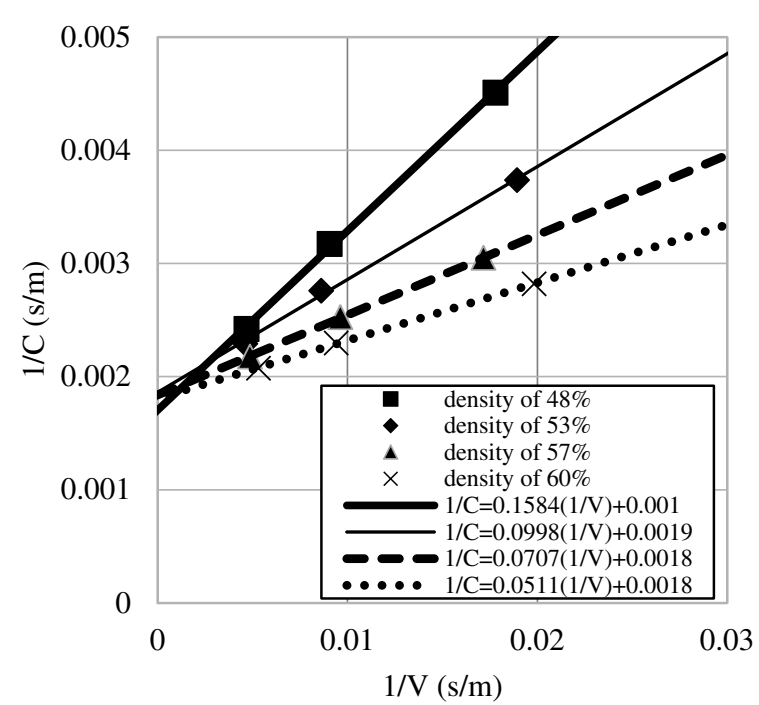

Figure 5. Relations (a), (b), (c), and (d) for packing density of $60 \%, 57 \%, 53 \%$, and $48 \%$, respectively.

target, the stress state near the projectile head region is not uniform due to the individual collision to the target particles as shown in (a), while such elastic state quickly spreads laterally and propagates upward almost alike unidirectional compression wave propagation as shown in (b). After the elastic wave reaches to the upper free end of the projectile, it reflects back as the tensile elastic wave and propagates down ward as shown in (c). Averaging the stress over the sectional area at the locations, A, and B, as indicated in the figure, the relations of the stresses at the A, and the B to time are shown in Fig. 7. It is apparently observed that the elastic wave reflections occur during penetration of the projectile. Assuming the elastic wave propagation without retardation and free end reflection on the upper end of the projectile, the force at the impact head can be evaluated by superposing the elastic waves detected at the locations A, and B, and is given in Fig. 8(a,b) for the projectiles of $120 \mathrm{~mm}$ and $200 \mathrm{~mm}$ in length. It should be noted that the force decreases after the peak value with periodical change corresponding to the reflection of stress wave as indicated by an arrow, since the periods of 48 and 80 microseconds for the projectiles of $120 \mathrm{~mm}$ and $200 \mathrm{~mm}$ in length, respectively. It is also apparent that the force evaluated from acceleration of the projectile well corresponds to the force obtained by stress wave analysis, even though significant undulations appear at around the peak value. The relations between reaction force and time are strongly influenced by impact velocity as shown in Fig. 9. Significant increase of peak value can be understood by change of momentum of particles in target as discussed before [6].

\subsection{Effect of mechanical properties of projectile}

Effect of mechanical properties of projectile on the resistance force during penetration was also investigated.

Based on the one-dimensional elastic wave propagation theory, the reaction force of the projectile can be evaluated as follows. Let's denote $\rho, C$, and $\rho_{t}, C_{t}$, are
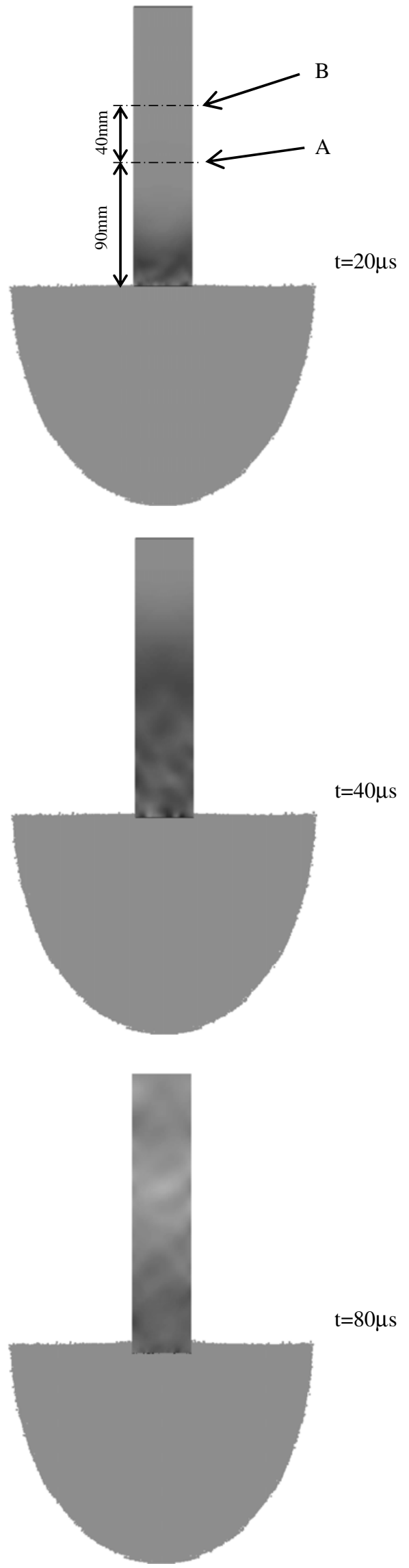

Figure 6. Distribution of axial stress in projectile in time. 

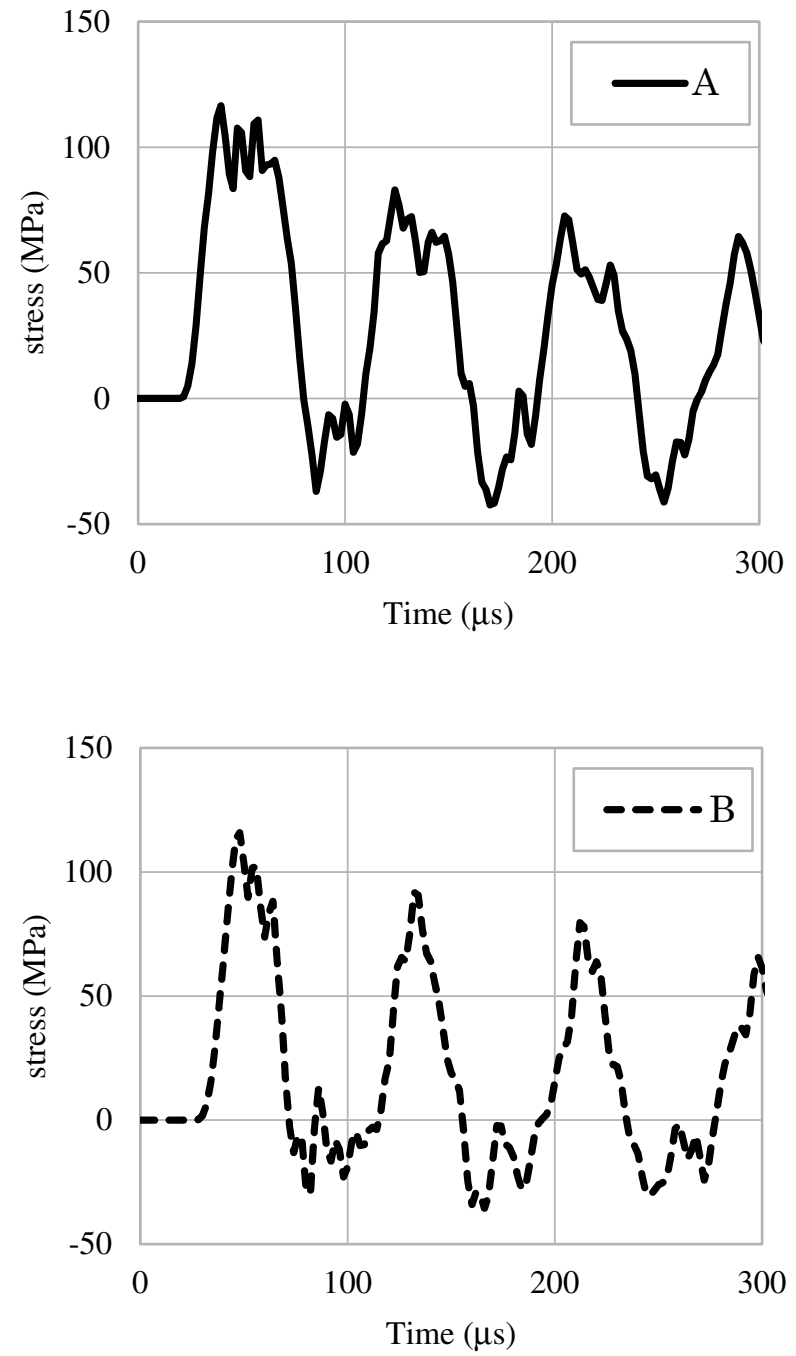

Figure 7. Stress waves detected at locations A, and B in a projectile.

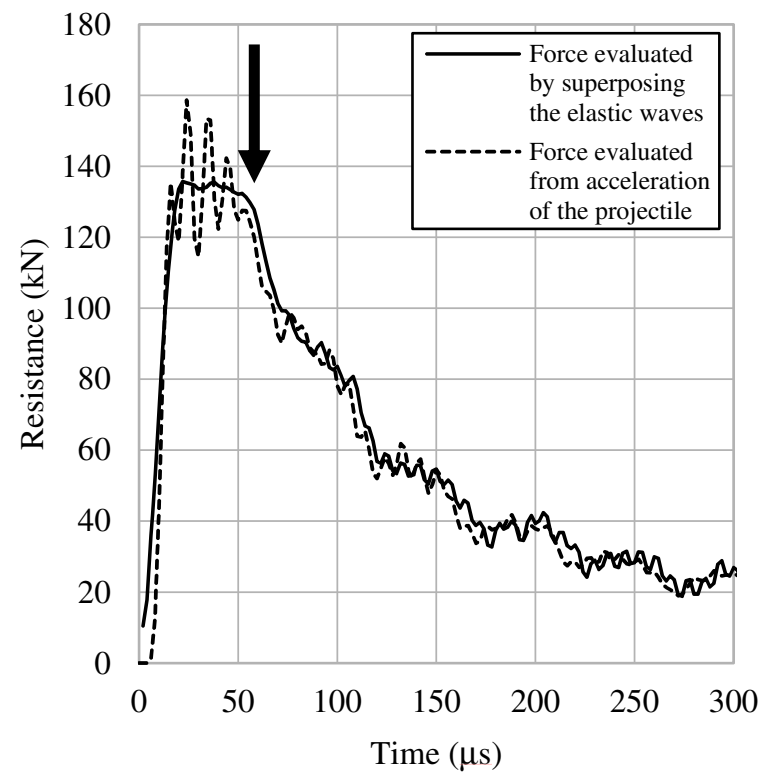

Figure 8. a) Force-time relations derived from stress wave detected in projectile of $120 \mathrm{~mm}$ in length.

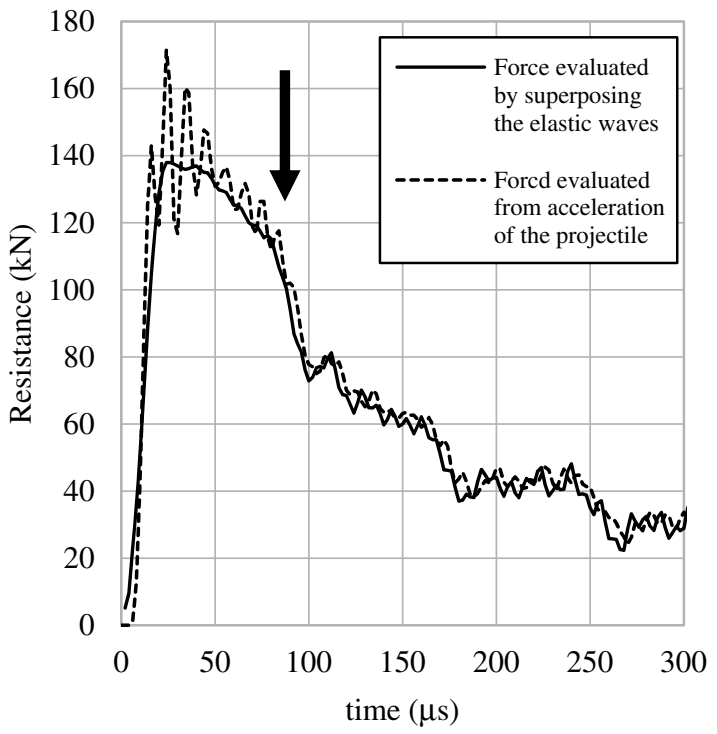

Figure 8. b) Force-time relations derived from stress wave detected in projectile of $200 \mathrm{~mm}$ in length.

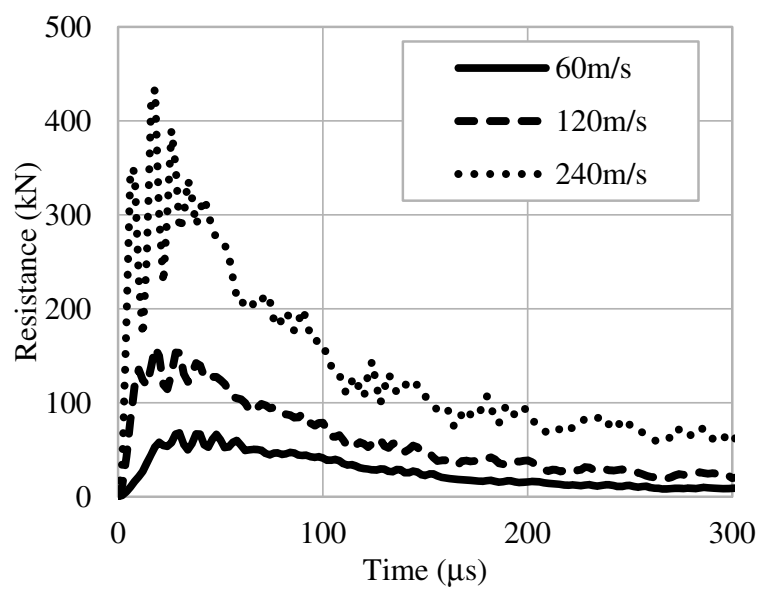

Figure 9. Force-time relations at various impact velocity.

the respective density and wave velocity of the cylindrical projectile and the target.

The reaction force $F$ at initial impact velocity of $V_{0}$ can be expressed as follows

$$
F=A_{0} \sigma_{T}=A \sigma_{R}=\frac{V_{0}}{\left(\frac{1}{\rho C A}+\frac{1}{\rho_{t} C_{t} A_{t}}\right)} .
$$

Where, $A$ and $A_{t}$ are the sectional area of projectile and target, and $\sigma_{R}$ and $\sigma_{T}$ are the reflected and the transmitted stress wave amplitude in the projectile and the target, respectively. The effective sectional area of target, A, can be estimated as before [6], and is almost constant independent of the impact velocity. On the other hand, elastic wave velocity of the target depends on the impact velocity as shown in Fig. 5.

Now, we can see the effect of mechanical properties of projectile upon the resistance force as shown in Fig. 10. For the projectile of initial values of Young's modulus of $69 \mathrm{GPa}$ and of density of $2.8 \times 103 \mathrm{Kg} / \mathrm{m}^{3}$, the 

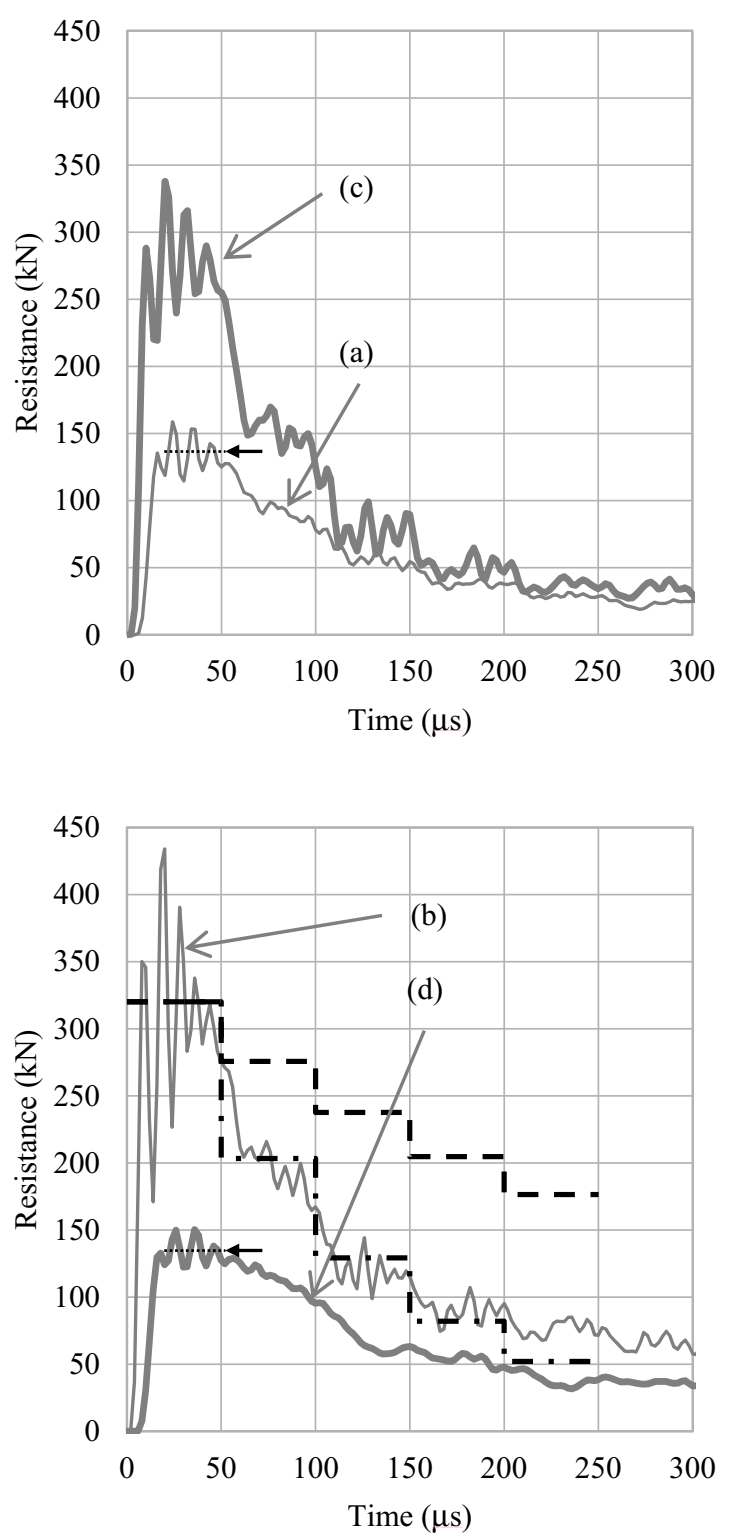

Figure 10. Effect of mechanical impedance and momentum of projectile on force-time curve.

force-time relations (a) and (b) are respectively obtained at initial impact velocities of $120 \mathrm{~m} / \mathrm{s}$ and $240 \mathrm{~m} / \mathrm{s}$. By reducing the Young's modulus and the density of the projectile to the half of their initial values the force -time relation shown by (c) is obtained at initial impact velocity of $240 \mathrm{~m} / \mathrm{s}$. The relation denoted by (d) is obtained at initial impact velocity of $120 \mathrm{~m} / \mathrm{s}$ for the projectile of the Young's modulus of half to the initial value and the density of twice the initial value. It is apparent from Eq. (4) that the value of reaction force $F$ is the same, when the value of $\rho C A$ is kept constant. Actually, almost identical values of initial reaction force $F$ indicated by the arrows on the curves (a) and (d) are obtained at the initial impact velocity of $120 \mathrm{~m} / \mathrm{s}$. It is very interesting that the momentum of projectile for curves (d) is twice of the momentum of projectile for curve (a), since the density for the former projectile is double of the latter. It is also found that the peak reaction forces for the curves (b) and (c) are different so far, even though the momentum is kept constant. From the peak reaction force, $F$, for the curves (a) and (b), the value of $\rho_{t} C_{t} A_{t}$ in the Eq. (4) can be evaluated as $1.19 \mathrm{KN} /(\mathrm{m} / \mathrm{s})$ and $1.43 \mathrm{KN} /(\mathrm{m} / \mathrm{s})$, respectively. Then, the peak reaction forces for the curves (d) and (c) can be estimated as $135 \mathrm{KN}$ and $299 \mathrm{KN}$, respectively. As shown in the figure, fairly good predictions are obtained. With taking account of dependence of the wave velocity $C_{t}$ in the target on the impact velocity as was mentioned before [6], evaluated values of $\rho_{t} C_{t} A_{t}$ at impact velocity of $120 \mathrm{~m} / \mathrm{s}$ and at $240 \mathrm{~m} / \mathrm{s}$ are still slightly different, but this can be understood by considering a possible change of $A_{t}$ by impact velocity already reported [6].

After the initial reaction, the stress wave in the projectile goes up and down and re-impact is continued to the target. Therefore, the same kind of equation like Eq. (4) is obtained for respective reverberations as follows;

$$
F_{i}=\frac{\left(1-\frac{\rho_{t} C_{t} A_{t}}{\rho C A}\right)}{\left(1+\frac{\rho_{t} C_{t} A_{t}}{\rho C A}\right)} F_{i-1} .
$$

Here, the reaction force after $i$-th reflection is represented as $F_{i}$.

In the figure, the relations obtained by Eq. (5) are represented by broken lines for the case of constant $\rho_{t} C_{t} A_{t}$, and by chained lines for the case of variable $\rho_{t} C_{t} A_{t}$. Judging from the results shown in Fig. 3, it would be reasonable to taking account of increase of $A_{t}$, since highly compressed region grows with penetration.

\section{Concluding remarks}

Three dimensional dynamic FEM and DEM simulations on the projectile penetration into granular target were performed and the followings were concluded.

(1) Propagation of elastic compression wave generated by penetration was observed and the dependence of wave velocity on density of particulate medium was clarified by means of simple expression.

(2) Periodical undulations on the reaction force curve were well understood in connection with propagation of elastic wave in the projectile.

(3) Mechanical impedance of projectile plays a predominant role on collision phenomena as well as the mechanical properties of target materials.

\section{References}

[1] Allen, W.A., Mayfield, E.B. and Morrison H.L., Jour. App. Phy., 28, 370 (1957).

[2] Backman, M.E. and Goldsmith, W., Int. Jour. of Eng. Sci., 16, 1 (1978).

[3] Bazhenov, V.G., Bragov, A.M. and Kotov, V.L., Jour. App. Mech. Tech. Phy., 50, 1011 (2009).

[4] Hauver, G.E., Inter. Jour. of Eng. Sci., 16, 871 (1978).

[5] Zhang, Y., Master's thesis for Osaka University, Japan(2013).

[6] Ogawa, K., Takeda, S. and Kobayashi, H., Mech. Eng. Jour. Jap., 02, (2015), (to be published). 